\title{
What is the General Interest in the Study of Fundamental Forces?
}

\author{
Hans-Peter Morsch* \\ HOFF, Juelich, Germany
}

Submission: February 23, 2018; Published: May 22, 2018

*Corresponding author: Hans-Peter Morsch, HOFF, Brockmüllerstr. 11, D-52428 Jülich, Germany; Email: h.p.morsch@gmx.de

Abstract

In astrophysics many features of the universe can be studied, but no answer can be given on fundamental questions as: How has the universe been created? What was its structure before the "Big Bang"? The only possibility to access this regime is the study of fundamental forces between elementary particles. Since nature develops in a logic way, we expect to get clear answers to these questions from the correct theory of these forces.

Keywords: Astrophysics; Structure and development of the universe; Big Bang; Einstein's theory of general relativity; Fundamental forces; Crisis of Standard Models: Non-existence of super-symmetric particles; Breaking of matter-antimatter symmetry; Dark matter; Fundamental bound state theory without open parameters; Consistent description of hadrons and atoms by electric forces; leptons and gravitation by magnetic forces. Importance of chiral properties

\section{Short Communication}

The structure and development of the universe has been studied in detail with wide angle telescopes and extraterrestrial missions, using all spectroscopic tools available by modern detector technologies. But essentially all this information is related to the cosmic structure after the "Big Bang", from which the universe expanded from a small volume to the present gigantic size. What happened before escapes almost completely our observation. The expansion of the universe is described in the Standard Model of Astrophysics [1], which is based on Einstein's theory of general relativity [2]. According to this theory the universe arose from a singularity (or an extremely small volume, if a finite cut is introduced), but why it should have such a small size is not understood. Further, astrophysical observations require the assumption of a rapid inflation in space before the cosmic expansion started. Why all this happened is not explained and subject of speculative interpretations.

The only way to get serious answers on these questions is a study of fundamental forces. In particle physics experiments we are able to study the interplay of elementary particles and their interactions, which have taken place in the early phase of the universe. Therefore, by the correct theoretical interpretation of high energy experiments an understanding of this cosmic regime should be possible. Unfortunately, the theoretical description in both Standard Models of Particle Physics and Astrophysics is presently in a severe crisis $[1,2]$. These models cannot provide a convincing physical picture of the cosmic development, the observed matter antimatter inequality of the universe, the non- observation of super symmetric particles as well as the predicted dominance of dark matter and dark energy in the universe.

These problems appear to be related to the empirical construction of these Standard theories. For the four fundamental forces, electromagnetic, strong and weak forces as well as gravitation, the used theories are completely different. The first three forces, designed to understand the microscopic structure of matter, are described by quantum field theories, whereas the presently accepted theory of gravitation is Einstein's theory of general relativity, a classical theory, in which curved space-time is considered. Efforts to develop a quantum theory of gravity have been made, a field theory similar to quantum electrodynamics, which fails to describe important aspects of gravitation, and string theories [3], which have promising features but an extremely complicated structure with many parameters. Attempts to find a unification of the different theories failed completely in the past.

What could be responsible for the above difficulties? Experimentally we know that particles have finite radii, and the interactions between particles are of finite range (this is also the case for the momenta). But all Standard theories are divergent for momenta $\rightarrow 0$ and $\infty$. Another serious shortcoming of these theories is the fact that external parameters are required, which cannot be deduced from basic boundary conditions. In the Standard Model of Particle Physics about 20 parameters are needed, coupling constants and masses of elementary fermions and bosons as well as their mixing. These parameters have been 
adjusted by experimental data. But in a really fundamental theory such external parameters should not be required (otherwise a more fundamental theory should exist, in which these parameters can be explained).

Only during the last years a fundamental bound state theory has been constructed [4,5], which is not divergent and has only a few parameters, which can be determined from basic boundary conditions. It is based on quantum field theory with a Lagrangian similar to that of quantum electrodynamics, but with fermions accompanied by boson fields. This theory has been applied first to the description of hadrons and atoms [4]. Surprisingly, the very different masses and radii of hadrons and atoms are described consistently by electric binding only, using three parameters (effectively one only), tuned to satisfy momentum as well as energy-momentum conservation. Further, within the same theory leptons are described by systems bound by magnetic forces [5]. Interestingly, this is the first theoretical description, in which the chiral structure (handedness) of leptons (left-handedness of electrons, right-handedness of positrons) could be explained.

Another important aspect of this theory is that a magnetic bound state of (e-p)-pairs has been found with an extremely small binding energy and a coupling constant in agreement with Newton's gravitational coupling constant $\mathrm{G}_{N} \sim 10^{-38}$. This indicates that gravitation can be described by magnetic binding of a large number of (e-p) pairs [5]. In the meantime the rotation of galaxies has been successfully analysed in this theory, with galaxy masses obtained by magnetic binding of about $10^{\wedge} 100$ (e-p) pairs [6]. (e-p) pairs. From additional constraints even information on the high density cosmic phase preceding the "Big Bang" could be extracted. This fundamental theory has promising features, which may allow us to answer all open questions. In this formalism there are no super-symmetric and other exotic particles. Further, there is no need to assume the existence of dark matter and dark energy, for which no real experimental evidence exists. Finally, this theory will allow us to get a realistic view of the early phase of the universe with the creation of matter, accumulation of the complete mass of the universe followed by a chirally controlled collapse and annihilation of half of the accumulated mass, by which the matter-antimatter symmetry has been broken.

\section{References}

1. Nakamura K (2010) Review of particle properties. J Phys G 37: 075021.

2. Einstein A (1923) Uber die spezielle und allgemeine Relativitats theorie. Z Phys 16: 228.

3. Aharony O, Gubser SS, Maldacena J, Ooguri H, Oz Y (2000) Large N Field Theories, String Theory and Gravity. Phys Rep 323: 183.

4. Morsch HP (2016) Brit J Math and Comp Sc 17(6): 1-11

5. Morsch HP, Ghosh S (2017) J Adv Math and Comp Sc 24(4): 1-11

6. Morsch HP (2018) Rotation of galaxies. Global J Science Front Res A 1(4): 18.

\section{Your next submission with Juniper Publishers} will reach you the below assets

- Quality Editorial service

- Swift Peer Review

- Reprints availability

- E-prints Service

- Manuscript Podcast for convenient understanding

- Global attainment for your research

- Manuscript accessibility in different formats

( Pdf, E-pub, Full Text, Audio)

- Unceasing customer service

Track the below URL for one-step submission https://juniperpublishers.com/online-submission.php 